\title{
Experimental study of gas phase titanium and aluminum oxide clusters
}

\author{
K. Demyk ${ }^{1,2}$, D. van Heijnsbergen ${ }^{1}$, G. von Helden ${ }^{1}$, and G. Meijer ${ }^{1,3,4}$ \\ 1 FOM-Institute for Plasmaphysics Rijnhuizen, Edisonbaan 14, 3439MN Nieuwegein, The Netherlands \\ 2 Present address: PhLAM, Université des Sciences et Technologies de Lille, 59655 Villeneuve d'Ascq Cedex, France \\ 3 Dept. of Molecular and Laser Physics, University of Nijmegen, Toermooiveld 1, 6525 Nijmegen, The Netherlands \\ ${ }^{4}$ Fritz-Haber-Institut der Max-Planck-Gesellschaft, Faradayweg 4-6, 14195 Berlin, Germany
}

Received 24 July 2003 / Accepted 23 February 2004

\begin{abstract}
We present an experimental study of the vibrational properties of gas phase titanium oxide and aluminum oxide clusters. The titanium and aluminum oxide clusters have a stoichiometry of $\left(\mathrm{Ti}_{2} \mathrm{O}_{3}\right)_{x}-\left(\mathrm{TiO}_{2}\right)_{y}$ (with $(x, y)$ from $(2,4)$ to $(11,29)$ ) and AlO- $\left(\mathrm{Al}_{2} \mathrm{O}_{3}\right)_{n}(5 \leq n \leq 70)$. The vibrational properties of the clusters are obtained using infrared resonance enhanced multiphoton ionization (IR-REMPI) spectroscopy. Titanium oxide clusters have a strong vibrational band at $\sim 13.5 \mu \mathrm{m}$, suggesting that their structure is close to the rutile bulk phase of $\mathrm{TiO}_{2}$. Aluminum oxide clusters seem to have a structure comparable to the bulk $\gamma-\mathrm{Al}_{2} \mathrm{O}_{3}$; their IR-REMPI spectra exhibit a vibrational band at $\sim 11 \mu \mathrm{m}$ and another band at $\sim 15 \mu \mathrm{m}$ which appears in the spectra of clusters containing more than 7-8 $\mathrm{Al}$ atoms and becomes more intense as the cluster size increases. As hot neutral clusters are observed to evaporate more easily electrons than neutral fragments, one can conclude that they are very stable and thus very good nucleation seeds for dust growth.
\end{abstract}

Key words. methods: laboratory - ISM: dust, extinction

\section{Introduction}

The presence of large organic molecules and/or very small particles (nanometric in size) in many and diverse astrophysical environments such as reflection and planetary nebulae, in the Interstellar Medium (ISM) or in galaxies is now well established thanks to space-born observations. For example, IRAS observations of an excess of emission at 25 and $60 \mu \mathrm{m}$ in the ISM have revealed the presence of very small grains (VSG) heated by the absorption of single UV photons (Désert et al. 1990). The exact composition of these grains is not yet known. The existence of various populations of nanograins has recently been proposed to explain various spectroscopic observations. The Extended Red Emission (ERE) observed in stars, planetary nebulae and in the ISM is proposed to be due to a population of nanograins of silicon (Ledoux et al. 2001; Witt et al. 1998) or nanograins of carbonaceous matter (Duley 2001). Nanodiamonds and titanium carbide nanoparticles have been proposed to be the carrier of the $21 \mu \mathrm{m}$ emission band observed in planetary nebulae (Hill et al. 1998; von Helden et al. 2000a).

Nanoparticles, containing few tens of atoms, are also produced around evolved stars. These grains are thought to play a crucial role in the dust formation process by constituting the nucleation seed on which bigger grains - such as silicate and

Send offprint requests to: K. Demyk,

e-mail: karine.demyk@univ-lille1.fr carbonaceous grains - will grow (e.g. Gail \& Sedlmayr 1999; Kozasa \& Sogawa 1997). Depending on the types of star, i.e. on the composition of their atmosphere, models of the dust formation process show that several nucleation seeds can be produced such as $\mathrm{Al}_{2} \mathrm{O}_{3}, \mathrm{TiO}_{2}, \mathrm{TiC}$ and/or $\mathrm{ZrC}$ clusters (Jeong et al. 1999; Ferrarotti \& Gail 2002).

Important efforts are made in the laboratory to study the physical properties of these "exotic" and poorly known species under conditions which approach those in the interstellar medium (see, for example, Spectrochimica Acta A 2001). Among these properties, vibrational frequencies are particularly important since these allow us to compare the experimental data to the astronomical observations.

In this paper we present an experimental study of the vibrational properties of gas-phase clusters relevant to the formation of interstellar dust: titanium and aluminum oxide clusters. The vibrational properties of the clusters are obtained using IR Resonance Enhanced Multi-Photon Ionization (IR-REMPI) spectroscopy (von Helden et al. 2003). In this technique, neutral clusters are irradiated with intense tunable IR radiation. When the IR radiation is resonant with an IR allowed vibrational mode of the cluster, multiple absorption of photons can occur, resulting in a dramatic increase in the internal energy of the cluster. These heated clusters may then dissociate or emit an electron via thermo-ionic electron emission. The latter only occurs for strongly bound clusters having a relatively low ionization potential. 
Infrared spectra of individual clusters are obtained by recording mass spectra and monitoring the intensity of the mass peak of the cluster of interest as a function of the IR wavelength. This technique has been successfully applied to a wide range of species such as fullerenes (von Helden et al. 1997), metal carbides (van Heijnsbergen et al. 1999; von Helden et al. 2000a) and oxides (von Helden et al. 2000b; van Heijnsbergen et al. 2002, 2003).

This paper is organized as follow. The experimental setup and procedure are described in Sect. 2. The results are presented in Sect. 3. Discussion of the results and the astrophysical implications are presented in Sects. 4 and 5, respectively.

\section{Experiments}

The experimental set-up, described in detail in von Helden et al. (2003), is only briefly outlined here. The clusters are produced by pulsed Nd:Yag laser vaporization at $532 \mathrm{~nm}$ of rotating metal rods ( $\mathrm{Ti}$ or $\mathrm{Al}$ ). The produced plasma is quenched in a mixture of $5-10 \%$ of $\mathrm{O} 2$ in Ar in which the clusters form. The pulsed beam expands into vacuum. After passage through a skimmer the charged clusters are deflected out of the beam by static electric fields. Then the neutral clusters enter the interaction region with the ionization laser, situated between the plates of a time-of-flight (TOF) mass spectrometer. Different ionization sources can be used such as excimer lasers (ArF or $\mathrm{KrF}$ ) or an infrared tunable laser. The IR light source used in the experiments is the Free Electron Laser for Infrared eXperiments (FELIX) in Nieuwegein, The Netherlands (Knippels et al. 1995). It is tunable from $40-2000 \mathrm{~cm}^{-1}$. In resonance with a vibrational transition of the cluster, the IR light can induce the excitation and ionization of this cluster. These ionized clusters are detected by TOF mass spectrometry. By varying the IR laser wavelength, IR-REMPI spectra are obtained.

\section{Results}

\subsection{Titanium oxide clusters}

Starting from a titanium rod and a pulsed flow of about 5-10\% of oxygen mixed with argon, titanium oxide clusters are produced by the method described in the previous section. The mass distribution of the clusters produced via IR-REMPI is obtained for different wavelengths of FELIX. The mass distribution spectrally integrated over the 9-24 $\mu \mathrm{m}$ wavelength range is shown in Fig. 1.

The mass spectrum is composed of groups of 3 mass peaks (see inset Fig. 1). The groups are equally spaced by $80 \mathrm{amu}$ corresponding to a $\mathrm{TiO}_{2}$ group. Within a group, the peaks are spaced by $16 \mathrm{amu}$ and the different peaks correspond to titanium oxide clusters having different oxygen content. Titanium has 5 naturally occurring isotopes. Consequently, the isotopic distribution of large titanium oxide clusters is very broad and their mass peaks overlap, causing the underlying continuum in the mass spectrum. Because the average titanium mass is three times the oxygen mass, it is difficult to unambiguously assign cluster stoichiometries to the mass peaks. In bulk $\mathrm{TiO}_{2}$, oxygen deficient structures are frequently observed and attributed

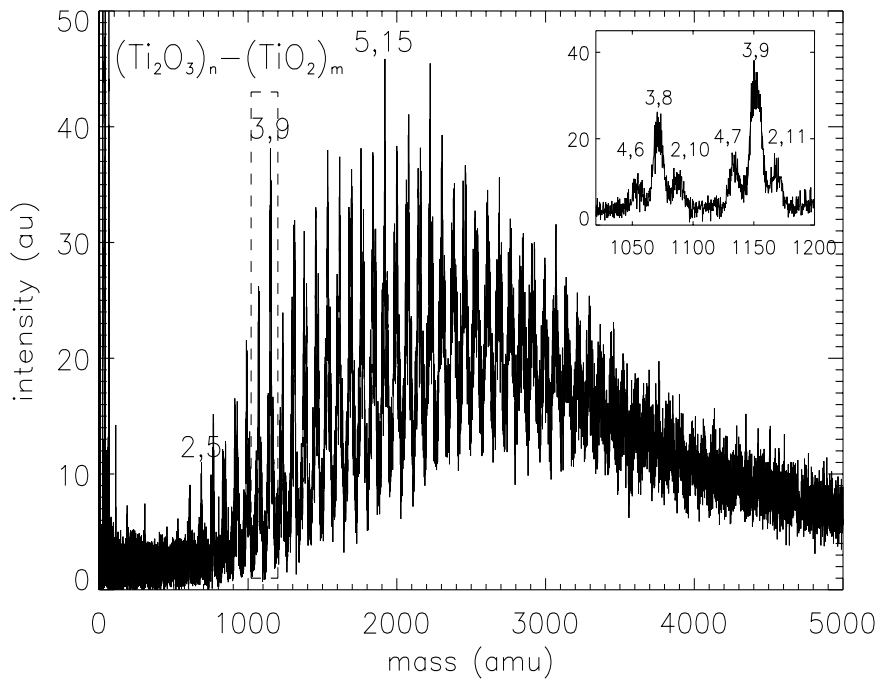

Fig. 1. Mass distribution of titanium oxide cluster cations produced via IR-REMPI. The mass distribution has been spectrally integrated over the 9-24 $\mu \mathrm{m}$ wavelength range.

to $\mathrm{Ti}_{2} \mathrm{O}_{3}$ sites. Furthermore, oxygen deficient clusters can be expected to have comparatively low IP's, being thus easier to detect in the IR-REMPI experiment. When looking at the low mass part of the cluster distribution, many possible combinations of $\mathrm{Ti}$ and $\mathrm{O}$ atoms can be excluded as assignments for mass peaks based on chemical arguments. A consistent assignment of the mass peaks starting from small clusters and following the observed progressions is that the clusters have a composition $\left(\mathrm{Ti}_{2} \mathrm{O}_{3}\right)_{x}-\left(\mathrm{TiO}_{2}\right)_{y}$ with $(x, y)$ from $(2,4)$ to $(11,29)$ (see von Helden et al. 2003 for further details). No dependence of the mass distribution on the incident IR beam intensity has been observed. This indicates that fragmentation processes are negligible and that the observed cluster ions are particularly stable against dissociation.

The wavelength of FELIX is tuned from 5 to $30 \mu \mathrm{m}$ with $0.1 \mu \mathrm{m}$ steps and a mass spectrum of the ionized clusters is recorded at each wavelength position. Plotting the intensity of a certain mass peak as a function of wavelength gives then its IR-REMPI spectrum. Spectra of different clusters are presented in Fig. 2 in the 9-24 $\mu \mathrm{m}$ range. In the 5-9 $\mu \mathrm{m}$ and $24-30 \mu \mathrm{m}$ wavelength ranges (not shown), no ion signal is observed. A Gaussian fit of the IR spectra shows that, as the size of the cluster increases, the position of the peak slightly shifts from $\sim 13.8$ to $\sim 13.5 \mu \mathrm{m}$ whereas the full width at half maximum ( $F W H M$ ) of the peak increases from $\sim 1.5$ to $3.8 \mu \mathrm{m}$. The spectra are thus similar for all cluster sizes and stoichiometries, suggesting that the structure of the clusters does not change dramatically as the clusters grow.

\subsection{Aluminum oxide clusters}

Aluminum oxide clusters are produced by ablating an aluminum rod in a $5-10 \% \mathrm{O}_{2}$ in argon mixture. Neutral clusters interact with FELIX and mass as well as IR-REMPI spectra are taken using the same methods as in the case of titanium oxide. A mass spectrum averaged over the wavelength range from $9 \mu \mathrm{m}$ to $18 \mu \mathrm{m}$ is shown in Fig. 3. Different to the case 


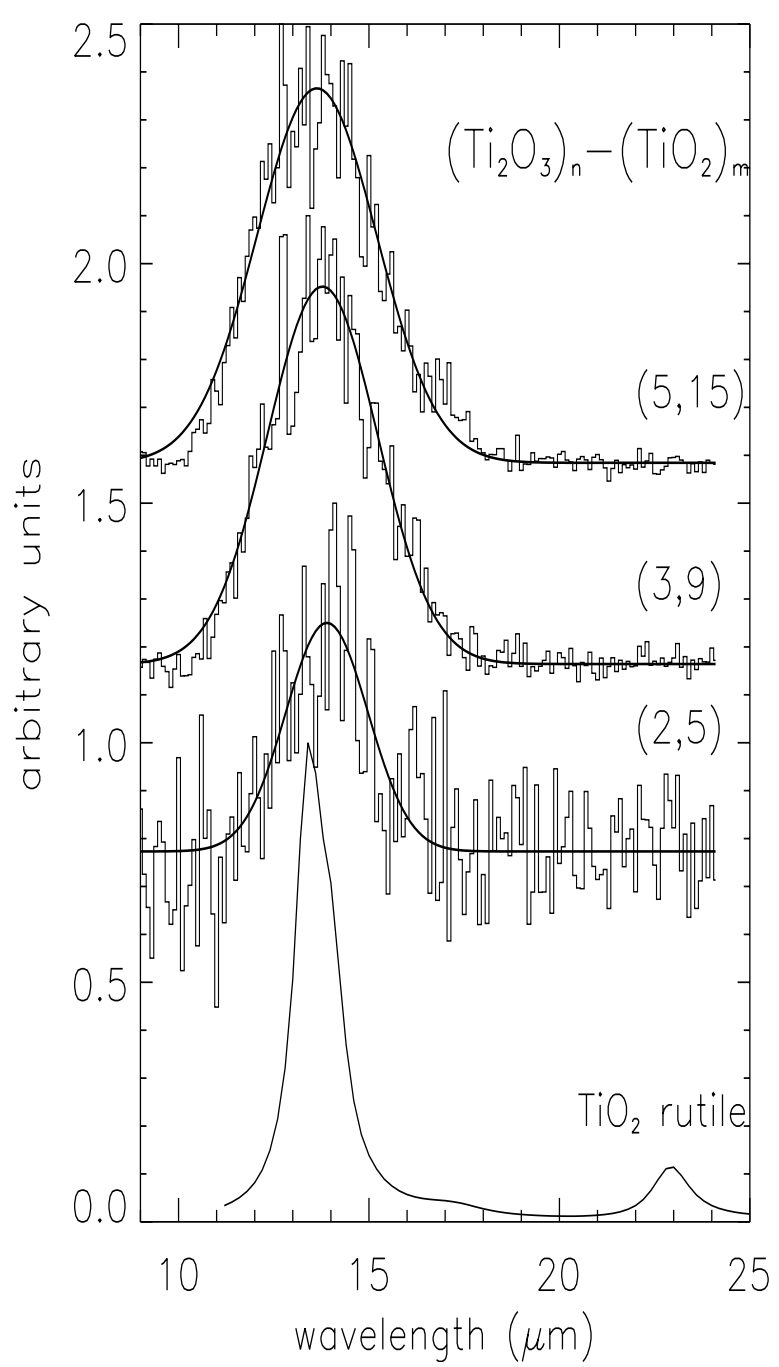

Fig. 2. IR-REMPI spectra of three titanium oxide clusters of different sizes and stoichiometries (histogram-like curve). The spectra have been shifted for clarity. From bottom to top the mass of the cluster is: $m=688,1152,1920 \mathrm{amu}$, with assignment: $\left(\mathrm{Ti}_{2} \mathrm{O}_{3}\right)_{2}-\left(\mathrm{TiO}_{2} \mathrm{O}\right)_{5}$, $\left(\mathrm{Ti}_{2} \mathrm{O}_{3}\right)_{3}-\left(\mathrm{TiO}_{2} \mathrm{O}\right)_{9},\left(\mathrm{Ti}_{2} \mathrm{O}_{3}\right)_{5}-\left(\mathrm{TiO}_{2} \mathrm{O}\right)_{15}$. The linear curves overlaying the experimental spectra represent Gaussian fits of the spectra. For comparison with the bulk phase, the bottom curve shows the extinction efficiency of rutile modeled from the optical constants for spherical grains in the Rayleigh limit (see Sect. 4.1).

of titanium, the mass of aluminum is not an integer multiple of the mass of oxygen and an unambiguous assignment of the mass peaks is possible. The clusters have the stoichiometry AlO- $\left(\mathrm{Al}_{2} \mathrm{O}_{3}\right)_{n}$ with $5 \leq n \leq 70$, close to that of bulk aluminum oxide. As in the case of titanium oxide clusters, the mass distribution of the aluminum oxides does not depend significantly on the intensity of the ionizing IR beam.

IR-REMPI spectra of aluminum oxide clusters are obtained by tuning the wavelength of FELIX in the $9-18 \mu \mathrm{m}$ range with $0.1 \mu \mathrm{m}$ steps. As can be seen in Fig. 4, the resulting infrared spectra of different clusters do depend on cluster size. The spectra of small clusters, with $n \leq 8-9$, show a prominent feature at $\sim 11 \mu \mathrm{m}$. As the cluster size increases, a band at $\sim 15 \mu \mathrm{m}$

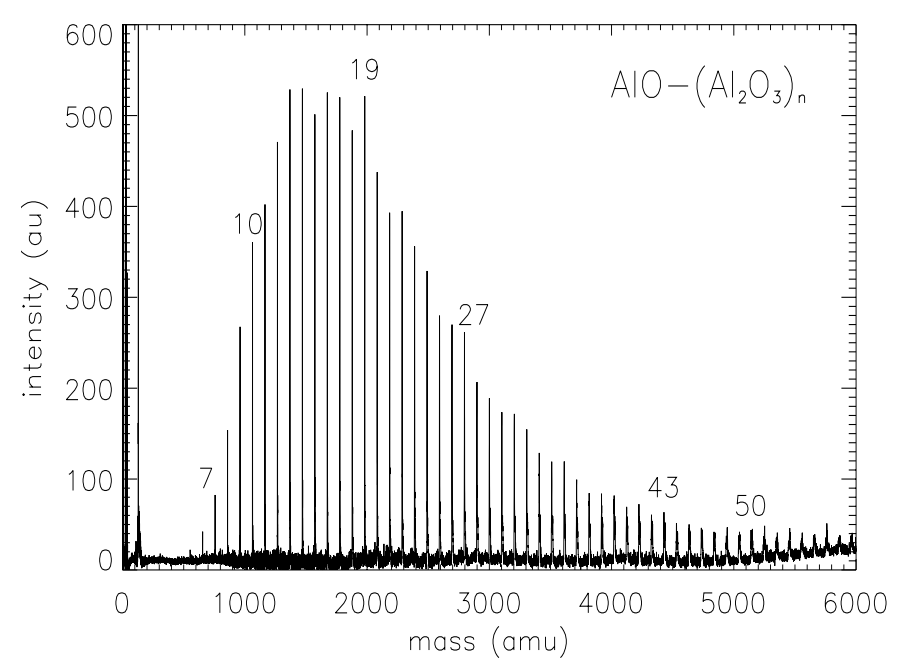

Fig. 3. Mass distribution of aluminum oxide cluster cations produced via IR-REMPI. The mass distribution has been spectrally integrated over the FELIX wavelength range of $9-18 \mu \mathrm{m}$.

appears in the IR spectra and its relative intensity with respect to the $11 \mu \mathrm{m}$ feature increases. In the IR spectra of the largest clusters produced in the present experiments, the two peaks have about the same intensity.

\section{Discussion: Comparison with bulk data}

\subsection{Titanium oxide clusters}

To our knowledge, no experimental vibrational data on gasphase titanium oxide clusters are available yet. However, electronic and structural properties of neutral titanium oxide molecules $\left(\mathrm{Ti}_{1-6} \mathrm{O}_{1-12}\right)$ have been investigated using density functional methods (Jeong et al. 2000). Depending on the stoichiometry and size of the molecules, Jeong et al. found IR transitions at $\sim 900-1000 \mathrm{~cm}^{-1}(10-11 \mu \mathrm{m})$ and/or at $\sim 600-800 \mathrm{~cm}^{-1}(12.5-16 \mu \mathrm{m})$. Results from these calculations show that, as the size of the molecules increases, the transition at $\sim 1050 \mathrm{~cm}^{-1}$ weakens; its intensity is less than $10 \%$ of the intensity of the $600-800 \mathrm{~cm}^{-1}$ bands for $\left(\mathrm{TiO}_{2}\right)_{n}$ clusters with $n \geq 5$.

Bulk titanium oxide can have different stoichiometries: $\mathrm{TiO}, \mathrm{TiO}_{2}, \mathrm{Ti}_{2} \mathrm{O}_{3}$ and $\mathrm{Ti}_{3} \mathrm{O}_{5}$. The most common, titanium dioxide, has three polymorphes: brookite and anatase which convert at high temperature into the third and most widespread type of titanium dioxide: rutile. The infrared spectra of rutile and anatase submicronic grains $(40-50 \mathrm{~nm})$, measured by Koike \& Shibai (1998), reflect the different structures of these two materials. The rutile spectrum shows a broad and strong band at $\sim 13-16 \mu \mathrm{m}$ and two weak bands around 25 and $29 \mu \mathrm{m}$. The spectrum of anatase shows two strong and broad bands around 17 and $29 \mu \mathrm{m}$.

Independently of their size and stoichiometry, the titanium oxide clusters have a vibrational transition at $\sim 13.5 \mu \mathrm{m}$. Although the clusters studied here are larger than those studied by Jeong et al. (2000), the position of the IR transition is in reasonable agreement with calculations on their largest molecules. More surprisingly, it is also in good agreement with the band 


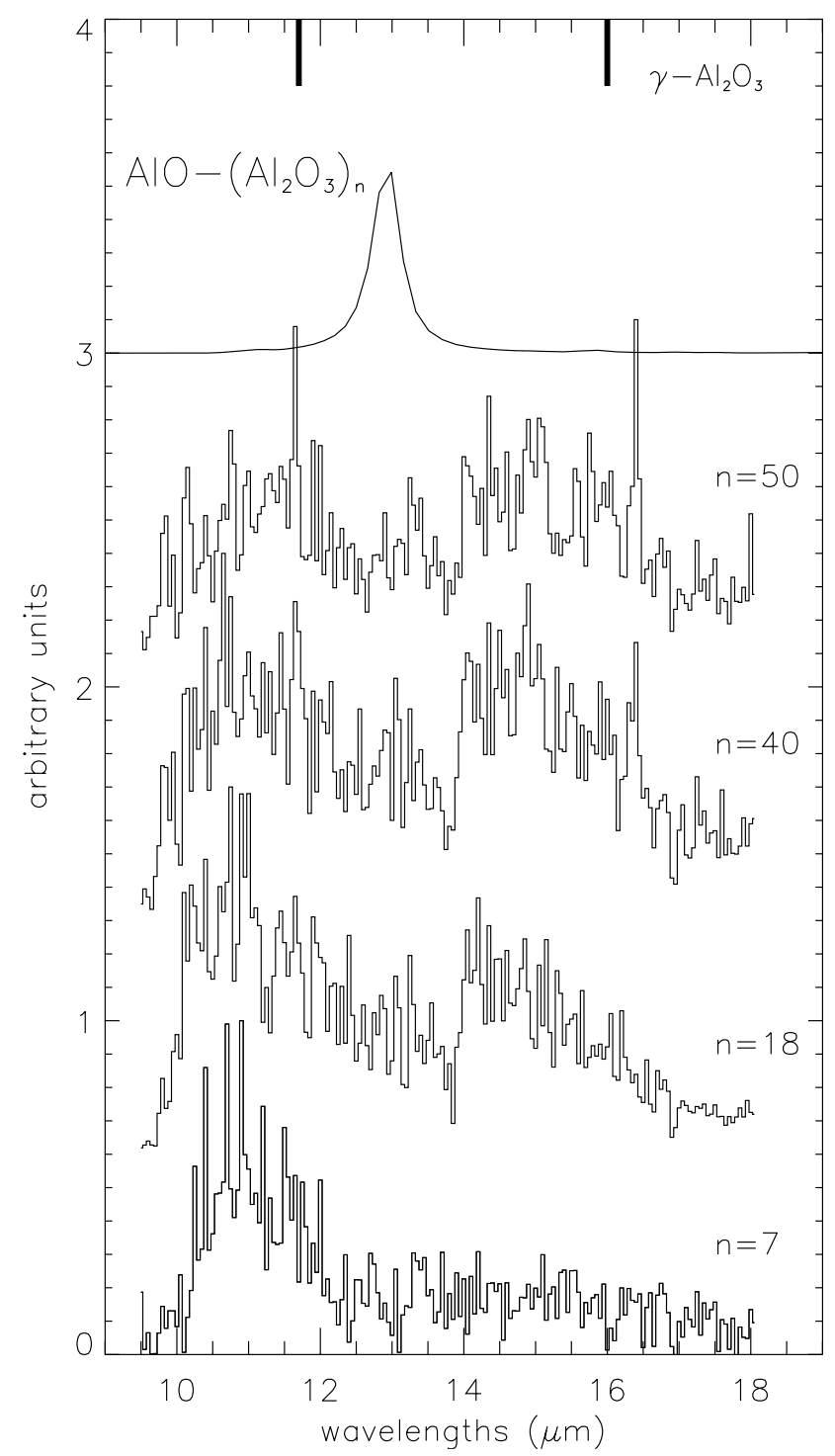

Fig. 4. IR-REMPI spectra of aluminum oxide clusters of different sizes. All spectra have been normalized to unity and shifted for clarity. The extinction efficiency of bulk $\alpha-\mathrm{Al}_{2} \mathrm{O}_{3}$ spherical grains, modeled from the optical constant (upper trace) and the position of the infrared bands of ultra thin films of $\gamma-\mathrm{Al}_{2} \mathrm{O}_{3}$ are shown for comparison (see Sect. 4.2).

position in the spectrum of bulk rutile grains (Koike \& Shibai 1998). This is also illustrated in Fig. 2 which shows the comparison between the spectrum of the titanium oxide clusters and the modeled spectrum of the bulk rutile $\mathrm{TiO}_{2}$ phase. The spectrum is modeled from the optical constants (Ribarsky 1985) using Mie Theory (Bohren \& Huffman 1983) for spherical grains in the Rayleigh limit (i.e. small with respect to the wavelength). The peak position of the observed vibrational band in the cluster spectrum is close to the one of rutile $(13.5 \mu \mathrm{m})$.

The agreement between the spectra of gas-phase clusters and rutile is not complete and two differences remain: the absence of the long wavelength bands (22.9 and $27.4 \mu \mathrm{m})$ observed in the rutile spectrum and the width of the $13.5 \mu \mathrm{m}$ band. The absence of the long wavelength bands in the cluster spectra could be due to experimental effects. For the IR-REMPI process to occur, a large amount of energy needs to be absorbed by the cluster. This means that when the wavelength gets longer, more and more photons need to be absorbed to reach the same internal energy. A combination of lower transitions strengths, anharmonicities and reduced laser fluence might thus makes it more difficult to observe transitions at longer wavelengths. The width of the peak is influenced by several factors. First, the spectrum is not a single photon absorption spectrum and the width of a multiphoton spectrum is influenced by the photon absorption dynamics (von Helden 2003). Additionally, the symmetry of the cluster will affect the width of the transition such, that for lower symmetry species, resonances will split and the result will be a peak that could consist of several unresolved resonances. Further, we cannot exclude an inhomogeneous broadening caused by the presence of several structural isomers that all have slightly shifted peak positions.

\subsection{Aluminum oxide clusters}

The infrared spectra of the aluminum oxide clusters change as the cluster size increases (Fig. 4). For very small clusters $(n \leq 8-9)$ only one band is observed at $11 \mu \mathrm{m}$. A band at $15 \mu \mathrm{m}$ appears in the spectrum of bigger clusters and becomes more prominent as the cluster size increases. Figure 4 shows the spectra of four $\mathrm{Al}_{2} \mathrm{O}_{3}$ clusters together with the modeled spectrum of crystalline bulk $\alpha-\mathrm{Al}_{2} \mathrm{O}_{3}$. As for the simulation of the absorption efficiency of titanium oxide, the spectrum of $\alpha$-aluminum oxide is modeled using Mie theory for spherical grains in the Rayleigh limit. The optical constants of $\alpha$-aluminum oxide were taken from Gervais (1991). Bulk $\alpha-\mathrm{Al}_{2} \mathrm{O}_{3}$ shows a strong band at $13 \mu \mathrm{m}$ and a weak band at $20.7 \mu \mathrm{m}$. Clearly the spectra do not match.

However, the comparison with IRAS and HREELS spectra of $\gamma-\mathrm{Al}_{2} \mathrm{O}_{3}$ ultra-thin films (Frank et al. 2001) is better. The spectrum shows a strong band at $11.5 \mu \mathrm{m}$ and two weak bands at 16 and $16.5 \mu \mathrm{m}$ (Fig. 4). These latter bands are probably not resolved in the IR-REMPI spectra in which only one band is observed at $\sim 15 \mu \mathrm{m}$. Other very weak bands at wavelengths greater than $20 \mu \mathrm{m}$ are observed in the spectra of thin films but are not present in the IR-REMPI spectra.

From these comparisons we conclude that the $\mathrm{Al}_{2} \mathrm{O}_{3}$ clusters have a structure close to the $\gamma-\mathrm{Al}_{2} \mathrm{O}_{3}$ bulk structure. This was also observed for nanometer-sized $\mathrm{Al}_{2} \mathrm{O}_{3}$ clusters $(5 \mathrm{~nm})$ produced from an $\alpha$ alumina sputter target (Ying et al. 1993). However, it is not clear if this is the case for bigger cluster $(n \geq$ 56-70). Depending on the experimental conditions, such clusters can also be produced. For these very large clusters, it is observed that the 11.5 and $16 \mu \mathrm{m}$ bands evolve into a very broad asymmetric band in the $10-20 \mu \mathrm{m}$ region. This band compares well with the modeled spectrum of spherical grains of amorphous $\mathrm{Al}_{2} \mathrm{O}_{3}$ (van Heijnsbergen et al. 2003). The fact that the structure of the $\mathrm{Al}_{2} \mathrm{O}_{3}$ clusters tends towards the structure of bulk $\gamma-\mathrm{Al}_{2} \mathrm{O}_{3}$ and/or of amorphous $\mathrm{Al}_{2} \mathrm{O}_{3}$, is compatible with the study of Gutiérrez \& Johansson (2002) who found that the structure of amorphous alumina presents close similarity with the surface structure of $\gamma$ alumina. 


\section{Astrophysical implications}

The formation of dust in the envelopes around late-type stars has been extensively studied (e.g. Gail \& Sedlmayr 1999; Kozasa \& Sogawa 1997). It is thought that this is a two step process. In that scheme small solid particles are first formed by clustering of molecular gas-phase species. These first condensates constitute nucleation seeds for the formation of bigger grains which grow by accreting molecules from the gas phase onto these seeds. The formation of different metal oxide clusters around evolved stars depends on the composition of the molecular gas phase of the circumstellar shell. For example oxide clusters such as $\mathrm{TiO}_{2}$ and $\mathrm{Al}_{2} \mathrm{O}_{3}$ are the most expected ones to form around oxygen-rich AGB stars (Jeong et al. 1999). Very small $\mathrm{Al}_{2} \mathrm{O}_{3}$ grains (10-20 $\AA$ ) are also expected to be formed in the ejecta of type II supernovae (Kozasa et al. 1991; Todini $\&$ Ferrara 2001). Around S stars which are transition objects between O-rich and C-rich stars at the top of the Asymptotic Giant Branch, both oxide and carbide clusters could nucleate such as $\mathrm{TiO}_{2}, \mathrm{TiC}, \mathrm{ZrC}$ (Ferrarotti \& Gail 2002). The latter two are also expected to form around carbon-rich stars.

The formation of these species around AGB or red giants stars is supported by the discovery of presolar inclusions in meteorites (Nittler et al. 1997). Indeed presolar grains such as TiC, nanodiamonds, $\mathrm{Al}_{2} \mathrm{O}_{3}$, have been observed in meteorites. A presolar titanium oxide grain has also been tentatively identified (Nittler \& Alexander 1999).

Some of these oxides or carbides, if they condense from the gas phase at higher temperature than the other dust species, may constitute the nucleation seeds for the dust formation. This seems to be the case for titanium dioxide and aluminum oxide and thus, titanium and aluminum oxide clusters appear to be good candidates for dust nucleation seeds in oxygen-rich stars.

The detection method used in the experiments is based on the thermo-ionic emission properties of the clusters. When heated, the clusters preferentially emit an electron instead of evaporating atoms or small molecules. This unique property is only observed for very strongly bound species like fullerenes and some pure metal, metal oxide, metal nitride and metal carbide systems. As thermo-ionic electron emission is observed for titanium and aluminum oxide clusters, those species must be very stable and resilient to dissociation. This has important consequences for the dust formation process in circumstellar shells and makes aluminum and titanium oxide clusters very good candidates for the dust nucleation process. In addition, the fact that the clusters undergo thermo-ionic emission rather than dissociation when heated has consequences for the growth efficiency. In a normal growth reaction where two particles $\mathrm{A}$ and $\mathrm{B}$ react to form the transient $\mathrm{AB}^{*}$, the binding energy has to be either carried away by a third collision partner or the transient $\mathrm{AB}^{*}$ has to cool itself by radiation to form a stable $\mathrm{AB}$ molecule. As the density around stars and in the ISM is too low for collisions with a third body to occur, radiative association is usually the only mechanism for growth. Since emission at IR radiation is a slow process, most of the transiently formed $\mathrm{AB}^{*}$ molecules will dissociate back to reactants. When the transient $\mathrm{AB}^{*}$ can now emit an electron instead, it can form the stable ion $\mathrm{AB}^{+}$. This ion can then recapture an electron to form $\mathrm{AB}^{*}$ again, which then has another chance to radiatively cool itself or the electron will leave again. In any case, the complex will not dissociate back to reactants A + B. In general, thermo-ionic electron emission might greatly enhance growth rates of small particles and should be included in the modeling of dust growth for those species.

Clearly, the excitation mechanism influences the obtained spectra, which can therefore be different from the linear absorption spectra. Anharmonicities will broaden lines and tend to shift them to the red. In that sense, the spectra measured here are expected to be comparable to emission spectra of warm species. A detailed discussion of the excitation mechanism and the relevance of multiphoton spectra to astronomical observations can be found elsewhere (Oomens et al. 2003)

Direct observation of the spectroscopic signatures of the dust nucleation seeds would be the most favorable in the ideal situation in which the dust formation process has just begun and other components of the dust such as silicates are not yet formed. In that case, even if the abundance of these seeds is low, it should be possible to observe their emission. However, this is generally not the case and the nucleation seeds are usually deeply embedded into larger grains. In that case, the silicate emission dominates. Some stars surrounded by dust shells dominated by oxide dust rather than by silicates have recently been found (Posch et al. 2002). Their spectra present a very broad emission feature at $10-22 \mu \mathrm{m}$ that Posch et al. reproduce with a mixture of amorphous $\mathrm{Al}_{2} \mathrm{O}_{3}$ and $\mathrm{Mg}_{0.1} \mathrm{Fe}_{0.9} \mathrm{O}$ grains to account for maxima at $\sim 11 \mu \mathrm{m}$ and $19.5 \mu \mathrm{m}$, respectively. These types of star may represent very good objects to look for the spectroscopic signatures of nucleation seeds such as aluminum and titanium oxides. Apparently, titanium oxide is not needed to get a reasonable fit of the spectra. However, regarding the broadness of the observed band, the fit is not unequivocal and the presence of titanium oxide cannot be excluded.

\section{Conclusion}

We have presented an experimental study of gas-phase aluminum and titanium oxide clusters. The vibrational properties of the titanium oxide clusters, $\left(\mathrm{Ti}_{2} \mathrm{O}_{3}\right)_{x}-\left(\mathrm{TiO}_{2}\right)_{y}$, are very similar for all cluster sizes and stoichiometries. Their IR-REMPI spectra show a vibrational transition at $\sim 13.5 \mu \mathrm{m}$, very close to the frequencies observed for the corresponding oxide bulk phase, rutile $\left(\mathrm{TiO}_{2}\right)$. The vibrational properties of the aluminum oxide clusters, $\mathrm{AlO}-\left(\mathrm{Al}_{2} \mathrm{O}_{3}\right)_{n}$, change with the cluster size. The IR-REMPI spectra of small clusters $(n \leq 7-8)$ exhibit a band at $\sim 11 \mu \mathrm{m}$. As the size of the clusters increases, a band at $\sim 15 \mu \mathrm{m}$ appears and its intensity increases. Comparison with IR spectra of $\alpha$-and $\gamma-\mathrm{Al}_{2} \mathrm{O}_{3}$ shows that the structure of the clusters is clearly different from $\alpha-\mathrm{Al}_{2} \mathrm{O}_{3}$ and points toward a $\gamma-\mathrm{Al}_{2} \mathrm{O}_{3}$ type structure. The large width of the vibrational transitions observed in the IR-REMPI spectra could be due to size effects inducing a certain degree of distortion or disorder in the arrangement of the atoms.

The ability of titanium and aluminum oxide clusters to undergo thermo-ionic emission illustrates their high stability. They thus constitute very good potential nucleation seeds on which the dust can grow. However, direct observation of such 
very small clusters will be difficult as they should rapidly be embedded into bigger grains. Furthermore, as the spectra of clusters containing more than $\sim 7-8$ metal atoms are identical for all clusters and are in addition close to the spectrum of the corresponding bulk species, it would be difficult to determine the size of the clusters and to distinguish between clusters and big, submicronic grains.

Acknowledgements. This work is part of the research program of the "Stichting voor Fundamenteel Onderzoek der Materie" (FOM), which is supported financially by the "Nederlandse organisatie voor Wetenschappelijk Onderzoek" (NWO). Financial support from the EU IHP Research Training Network (Delayed Ionisation and Competing Cooling Mechanisms in Atomic Clusters) and from the ESA (external fellowship) is gratefully acknowledged.

\section{References}

Bohren, C. F., \& Huffman, D. R. 1983, in Absorption and Scattering of Light by Small Particles (New York: John Wiley)

Desert, F. X., Boulanger, F., \& Puget, J. L. 1990, A\&A, 237, 215

Duley, W. W. 2001, ApJ, 553, 575

Ferrarotti, A., \& Gail, H. P. 2002, A\&A, 382, 256

Frank, M., Wolter, K., Magg, N., et al. 2001, Surf. Sci., 492, 270

Gail, H. P., \& Sedlmayr, E. 1998, Chemistry and Physics of Molecules and Grains in Space. Faraday Discussions (London: The Faraday Division of the Royal Society of Chemistry), 109, 303

Gail, H. P., \& Sedlmayr, E. 1999, A\&A, 347, 549

Gervais, F. 1991, in Handbook of Optical Constants II, ed. E. Palik (Acad. Press.), 761

Gutiérrez, G., \& Johansson, B. 2002, Phys. Rev. B, 65, 104202

van Heijnsbergen, D., von Helden, G., Duncan, M. A., et al. 1999, Phys. Rev. Lett., 83, 4983

van Heijnsbergen, D., von Helden, G., Meijer, G., et al. 2002, J. Chem. Phys., 116, 2400 van Heijnsbergen, D., Demyk, K., Duncan, M. A., et al. 2003, Phys. Chem. Chem. Phys., 5, 2515

von Helden, G., Holleman, I., Knippels, G. M. H., et al. 1997, Phys. Rev. Lett., 5234, 79

von Helden, G., Tielens, A. G. G. M., van Heijnsbergen, D., et al. 2000a, Science, 288, 313

von Helden, G., Kirilyuk, A., van Heijnsbergen, D., et al. 2000b, Chem. Phys., 262, 31

von Helden, G., van Heijnsbergen, D., \& Meijer, G. 2003, J. Phys. Chem. A, 107, 1671

Hill, H. G. M., Jones, A. P., \& d'Hendecourt, L. 1998, A\&A, 336, L41

Jeong, K. S., Winters, J. M., \& Sedlmayr, E. 1999, in Asymptotic Giant Branch Stars, ed. T. Lebertre, A. Lèbre, \& C. Waelkens, IAU Symp., 191, 233

Jeong, K. S., Chang, Ch., Sedlmayr, E., et al. 2000, J. Phys. B, 33, 3417

Knippels, G. M. H., Mols, R. F. X. A. M., van der Meer, A. F. G., et al. 1995, Phys. Rev. Lett., 75, 1755

Koike, C., \& Shibai, H. 1998, ISAP Report, \# 671

Kozasa, T., Hasegawa, H., \& Nomoto, K. 1991, A\&A, 249, 474

Kozasa, T., \& Sogawa, H. 1997, Ap\&SS, 251, 165

Ledoux, G., Guillois, O., Huisken, F., et al. 2001, A\&A, 377, 707

Nittler, L. R., Alexander, C. M. O. D., Gao, X., et al. 1997, ApJ, 483, 475

Nittler, L. R., \& Alexander, C. M. O. D. 1999, in Lunar and Planetary Science XXX, abstract No. 204

Oomens, J., Tielens, A. G. G. M., Sartakov, B. G., von Helden, G., \& Meijer, G. 2003, ApJ, 591, 968

Posch, Th., Kerschbaum, F., Mutschke, H., et al. 2002, A\&A, 393, L7

Ribarsky, M. W. 1985, in Handbook of Optical Constants of Solids, 795

Spectrochimica Acta A 2001, Special issue on astrophysics and astrochemistry 57 , Issue 4

Todini, P., \& Ferrara, A. 2001, MNRAS, 325, 726

Witt, A. N., Gordon, K. D., \& Furton, D. G. 1998, ApJ, 501, L111

Ying, J. Y., Benziger, J. B., \& Gleiter, H. 1993, Phys. Rev. B, 48, 1830 